Musées, Patrimoine et Culture scientifiques et techniques

$174 \mid 2017$

novembre-décembre 2017

\title{
Une question de point de vue ou la grande illusion des dioramas au Palais de Tokyo
}

\section{Pierre-Antoine Gérard}

\section{OpenEdition Journals}

Édition électronique

URL : http://journals.openedition.org/ocim/1879

DOI : $10.4000 /$ ocim. 1879

ISSN : 2108-646X

\section{Éditeur}

OCIM

Édition imprimée

Date de publication : 1 novembre 2017

Pagination : $36-39$

ISSN : 0994-1908

Référence électronique

Pierre-Antoine Gérard, « Une question de point de vue ou la grande illusion des dioramas au Palais de Tokyo ", La Lettre de I'OCIM [En ligne], 174 | 2017, mis en ligne le 01 novembre 2018, consulté le 24 juillet 2019. URL : http://journals.openedition.org/ocim/1879 ; DOI : 10.4000/ocim.1879

Ce document a été généré automatiquement le 24 juillet 2019.

Tous droits réservés 


\title{
Une question de point de vue ou la grande illusion des dioramas au Palais de Tokyo
}

\author{
Pierre-Antoine Gérard
}

1 Quels points communs entre un extrait du film La nuit au musée (2006), Le martyre de SaintBarthelemy réalisé en cire polychrome par Ignacio Lo Guidice au début du XVIII ${ }^{\mathrm{e}}$ siècle, une rue de Paris reconstituée par l'artiste américain Mark Dion (2016) et une scène figurant deux chouettes Athena noctua naturalisées par Edwart Hart en 1916. Ces œuvres ou documents sont ou font référence aux dioramas.

2 Le Palais de Tokyo a présenté jusqu'au 9 septembre 2017 Dioramas, une grande exposition dédiée à ces dispositifs muséograhiques caractérisés par la présence d'objets tridimensionnels évoluant dans un décor reconstitué et isolés par une vitre ou une grille, bien connus dans les muséums d'histoire naturelle. Servie par une muséographie épurée qui place judicieusement dans la pénombre le visiteur comme élément étranger aux dioramas, qui sont, eux, mis en lumière, cette exposition rassemble plus d'une centaine d'œuvres et documents. Il convient de souligner l'originalité de la démarche du Palais de Tokyo de consacrer une exposition à ce seul médium muséographique. Riche, précise et pluridisciplinaire, elle replace les dioramas dans une perspective historique, depuis leurs origines avec les célèbres théâtres illusionnistes de Louis Daguerre (1787-1851) jusqu’à leur réinvestissement par des artistes contemporains.

3 Tantôt, instrument de propagation de la foi, tantôt de célébration de la nature, de propagande politique ou témoin ethnographique, les dioramas s'établissent entre art et science, statique et mouvement, illusion optique et matérialité spatiale. Longtemps très prisés du public pour leurs qualités didactiques, contemplatives et parfois oniriques, à une époque où le cinéma n'existait pas encore, ils disparurent peu à peu des salles d'expositions avant de se voir réappropriés par des artistes qui trouvent en ces dispositifs un merveilleux support pour élaborer une lecture critique de notre société.

4 Les dioramas d'histoire naturelle figurent parmi les présentations les plus spectaculaires de cette exposition. Ils ont peuplé les muséums au cours des XIXe et XXe siècles avant de 
progressivement disparaître pour laisser place à des muséographies d'une autre nature et des formes de médiation différentes. Destiné à "voir au travers", le diorama présentait au sein d'un décor reconstitué, une scène naturaliste, donnant l'illusion du mouvement grâce à des taxidermies adaptées. En France, on peut citer le plus ancien qui fut conçu au muséum de Rouen sur le thème de la ferme normande (1892) et l'éphémère musée du Duc d'Orléans (1928-1959). À la fois pédagogiques et contemplatifs, certains subsistent dans les muséums et notamment à Berlin, Ottawa, Neuchâtel, ou encore à New York, dans l'African Hall, que l'on doit au maître incontesté de la discipline : Carl Hakeley. L'exposition rend hommage à ses travaux, notamment ceux consacrés aux gorilles si chers à Hakeley, en présentant des photographies de ses dioramas new-yorkais. Sont également présentés les originaux des études de ses peintures de fonds panoramiques. Ces éléments constitutifs des dioramas contribuent significativement à l'extrême qualité des présentations de Hakeley qui demeurent, aujourd'hui encore, d'une redoutable efficacité !

\section{Walter Potter, Happy Family, vers 1870}

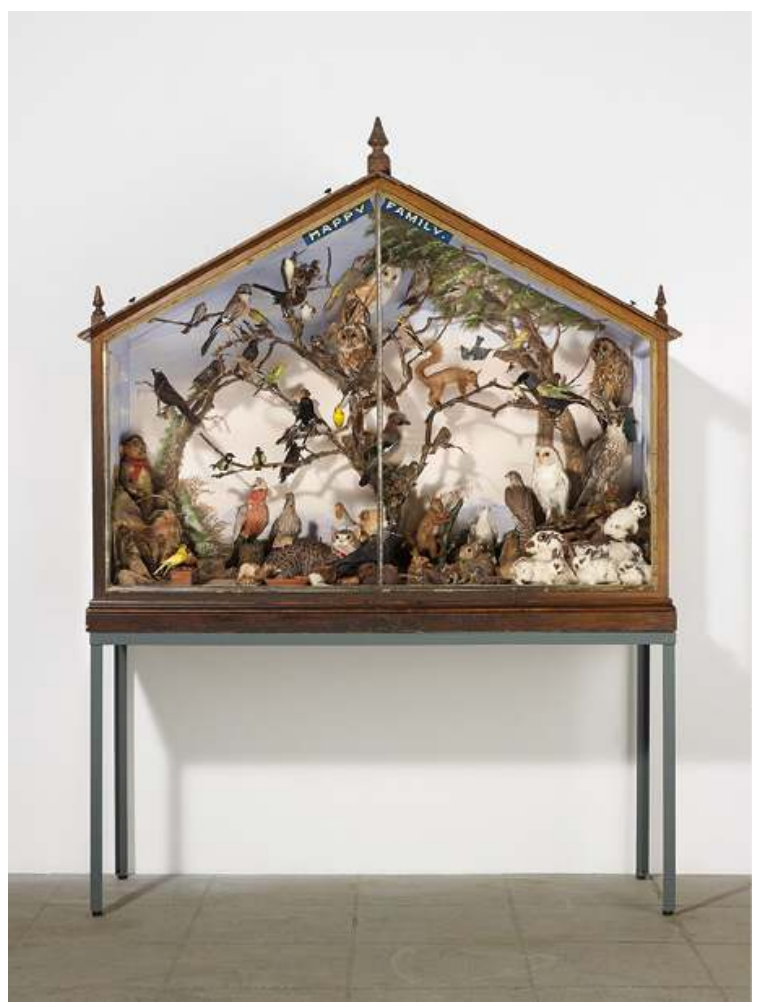

(C) PRUDENCE CUMINg ASSOCIATES

5 La place des dioramas d'histoire naturelle dans cette exposition est conséquente, avec la présence des réalisations délicates et précises d'Edwart Hart, ainsi que celles de Rowland Ward avec son impressionnant Léopard et guibs harnachés (1904). Le visiteur a également pu découvrir le surprenant et fantaisiste Happy family de Walter Potter (vers 1870). Ces dioramas sont judicieusement complétés par des photographies énigmatiques et fascinantes... de dioramas. En particulier celles de Robert Gober (1976-1977) et celles de Sugimto Sugimoto qui joue par une parfaite utilisation des contrastes, avec notre perception visuelle, sanctuarisant l'immobilité de la mort (séries réalisées entre 1977 et 2012 dans des muséums américains). 
Une courte section de l'exposition Voir à travers rassemble des œuvres d'artistes, tantôt dévoilant ce qui est habituellement invisible au visiteur, tantôt captant avec humour des irruptions du réel dans l'univers figé et romantique des dioramas. Les photographies de Richard Barnes ou de Diane Fox sont particulièrement touchantes à cet égard.

7 Loin d'être des dispositifs propres aux muséums d'histoire naturelle, les dioramas trouvent leur origine dans une longue tradition religieuse qui semble remonter aux crèches napolitaines de la Renaissance. L'exposition Dioramas met en lumière ces productions religieuses destinées à incarner le mystère de la foi et à favoriser la dévotion des fidèles. Plusieurs œuvres possédant les caractéristiques classiques des dioramas figurent ainsi des scènes bibliques comme le Calvaire napolitain de Lorenzo Mosvers (vers 1760-1780). Ces proto-dioramas, le plus souvent conçus en cire polychrome, figurent parmi les trésors de cette exposition de par l'exceptionnelle précision apportée aux personnages et décors de ces scènes reconstituées. On retrouve également des dioramas plus surprenants comme ceux reconstituant, en miniature, des intérieurs de cellules de carmélites datés du début du XIX ${ }^{\mathrm{e}}$ siècle.

Lorenzo Mosvers, Calvaire napolitain, vers 1760-1780

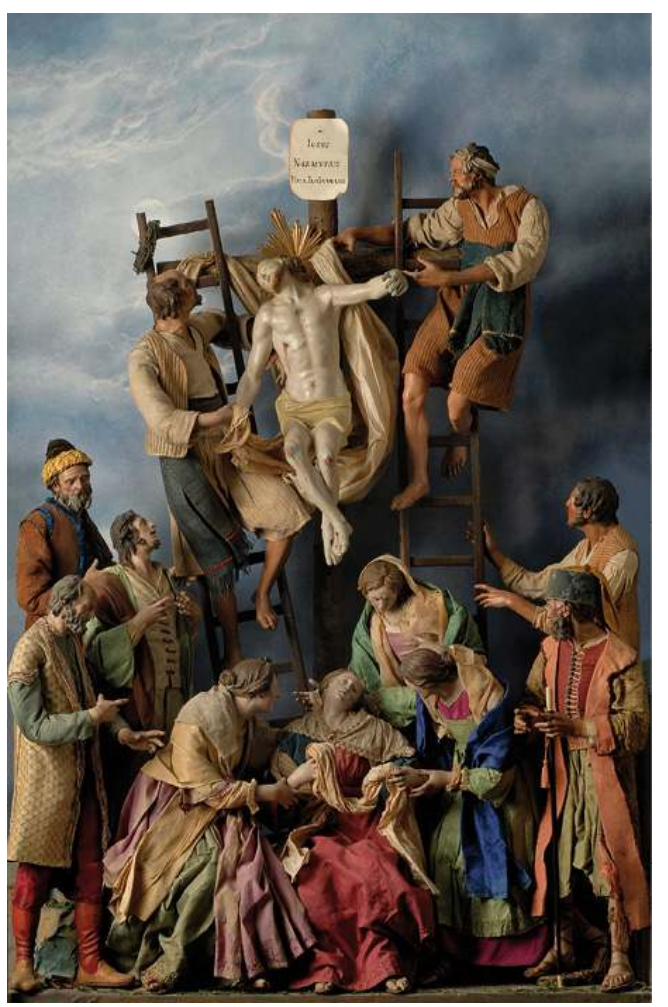

(c) Oreste Lanzetta

Avant cela, l'exposition avait ouvert sur la géniale invention de Louis Daguerre qui réalisa à Paris en 1822, un théâtre illusionniste permettant d'intégrer le mouvement à la peinture au moyen de toiles perforées et d'un savant jeu de lumières. Inspiré des panoramas, ces spectacles grandioses et en trompe l'œil de fresques romantiques ou historiques furent rapidement appelés dioramas et connurent un succès phénoménal. Véritable ancêtre du cinéma à venir, le monde forain s'empara de cette technique pour réaliser des présentations et spectacles à travers le monde, notamment aux États-Unis. L'exposition donne à contempler divers documents et œuvres témoins de ces "théâtres 
optiques" tout comme une représentation de la peinture que Louis Daguerre réalisa en 1842 en l'église de Brie-sur-Marne.

L'exposition consacre ensuite une importante section aux dioramas humains, fréquemment présentés dans les Expositions universelles ou coloniales du début du XX siècle comme celle de Nancy en 1909, de Stuttgart en 1928 ou de Paris en 1931. Représentations du pouvoir de l'être humain sur la nature, mais également sur ses congénères, le diorama a servi d'instrument de propagande politique et traduit la conquête coloniale et de douteuses théories raciales. L'exposition propose ainsi à voir plusieurs dioramas humains comme Légumes et fruits de Marcelle Ackein (1931) ou ceux du hollandais Gerrit Schouten, qui figurent des scènes de vie dans les colonies au début du $\mathrm{XX}^{\mathrm{e}}$ siècle. Ces dioramas, considérés comme des souvenirs folkloriques, participèrent par leur matérialité politique, à la diffusion des théories anthropologiques de l'époque tout en suscitant le sentiment idéalisé et exotique des co-lonies. La section est également complétée par des dioramas "préhistoriques" comme le méticuleux Culture de la chasse, Homo sapiens dans une grotte à l'âge de pierre de Fritz Laube (1960-1970) ou historiques par la représentation de batailles héroïques dans la Russie soviétique ou pour l'indépendance d'anciennes colonies comme le Vietnam ou les Philippines avec les œuvres de John Fontcuberta (2005).

Marcelle Ackein, Légumes et fruits, 1931

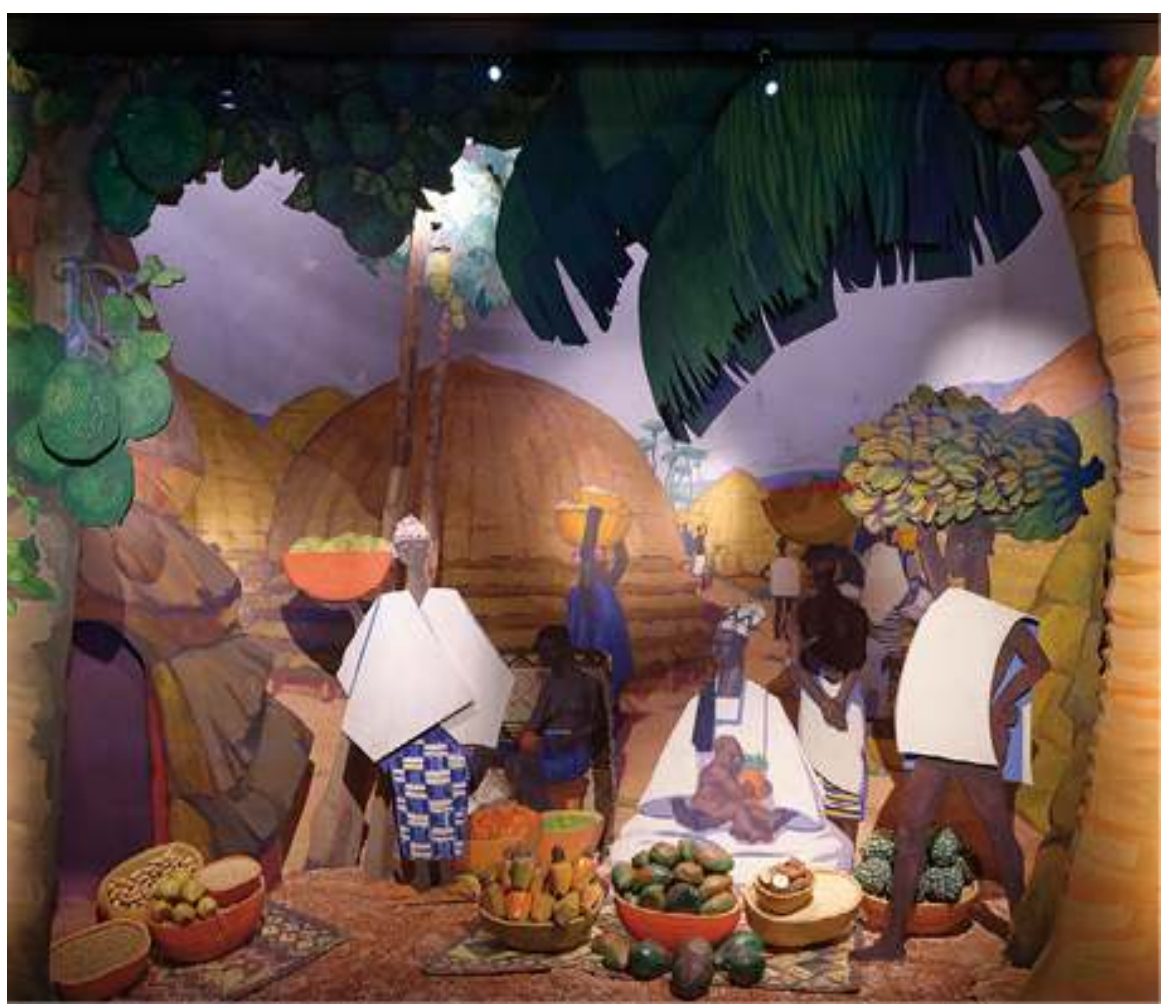

(c) RMN-Grand Palais / Photo J-G Berizzi

L'exposition s'intéresse également à l'ethnographie française, puisqu'elle rend hommage à l'œuvre de Frédéric Mistral mais aussi, et surtout, de Georges Henri Rivière (1897-1985) à qui l'on doit la révolution muséographique et scientifique menée dans le domaine des Arts et traditions populaires (ATP). La vitrine Du berceau à la tombe de l'ancien musée national des ATP, ouvert en 1937 à Paris, est reconstituée. Dans un contexte moderniste 
où l'on craignait la disparition des nombreuses traditions locales, ce mouvement qui visa à collecter les objets et témoignages du quotidien des régions de France, fut muséographié de manière à reconstituer, avec force rigueur scientifique, les intérieurs des habitations, ateliers... et appréhendés comme des "unités écologiques". Ce "magicien des vitrines" que fut Georges Henri Rivière intégra à ses dioramas des dispositifs lumineux et sonores favorisant l'immersion du visiteur. À l'instar des dioramas d'histoire naturelle, cette muséographie si caractéristique de l'œuvre de Georges Henri Rivière, tomba en désuétude dans les années 1980 provoquant la fermeture du musée puis, des années plus tard, le transfert d'une partie des collections au MuCEM à Marseille.

Georges-Henri Rivière, Du berceau à la tombe, Galerie culturelle du MnATP, 1975

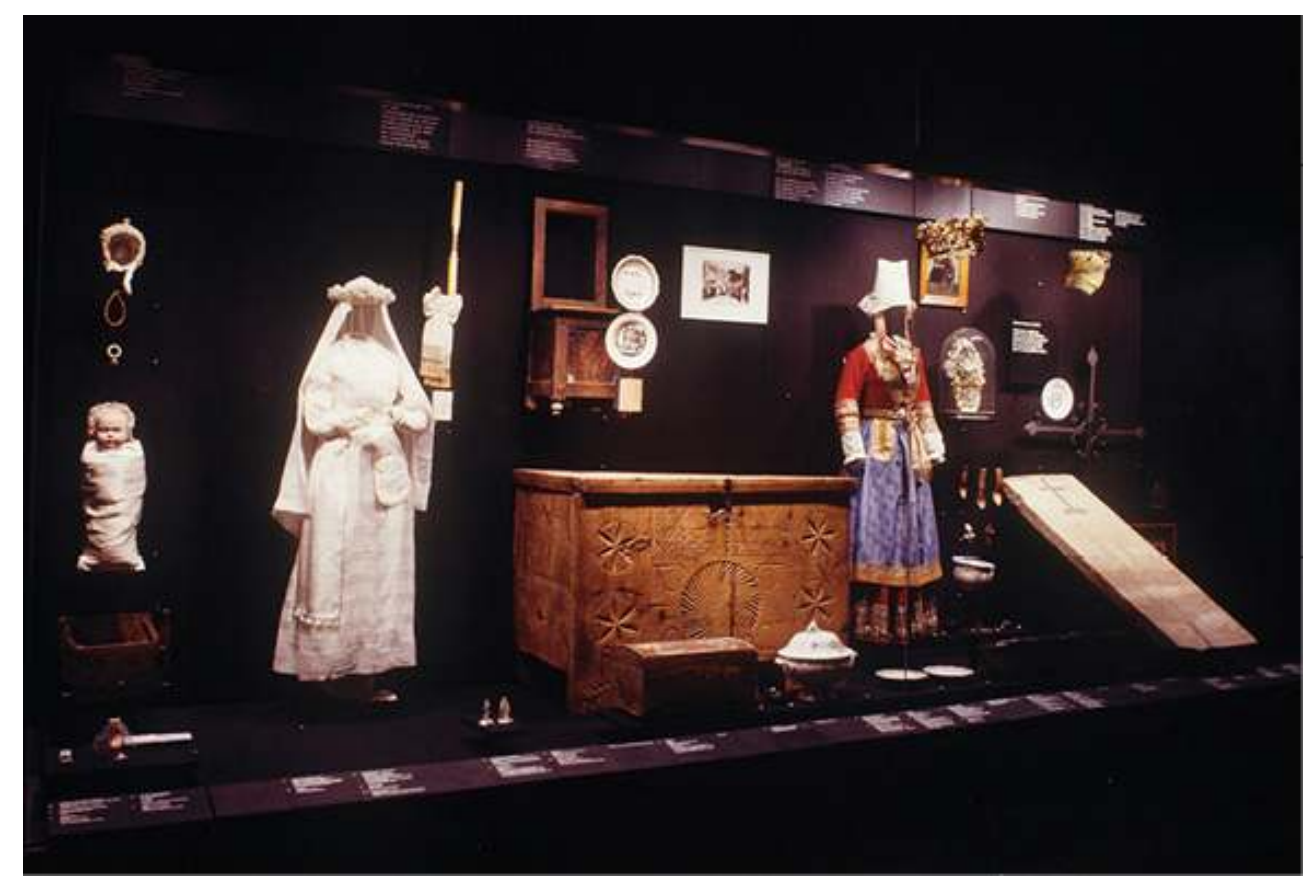

(c) Mucem / Hervé Jézéquel

11 La dernière section de l'exposition Dioramas présente aux visiteurs des œuvres, tantôt intimes, tantôt spectaculaires, créées entre la seconde moitié du XXe siècle et aujourd'hui. Elles témoignent des productions d'artistes qui réinvestissent, en même temps qu'ils détournent, les codes de ce dispositif muséographique pour analyser et porter un regard critique sur nos sociétés contemporaines. Les œuvres intimistes empreintes de mélancolie de Joseph Cornell, de Ronan-Jim Sévellec ou encore de Charles Matton entrent en résonnance avec des œuvres plus dérangeantes comme Family Pictures d'Anselm Kiefer (2013-2017) ou Étant donnés : $1^{\circ}$ la chute d'eau, $2^{\circ}$ le gaz d'éclairage... de Marcel Duchamp (1946-1966). Certaines œuvres sont davantage encore critiques, voire catastrophistes comme Hunting et collecting de Sammy Baloji (2015) qui pointe les abus des anciennes nations coloniales, la scène d'apocalypse Empire Vampire III, 12 d'Isa Genzken (2004) et encore le mystérieux Panorama 14, d'Armand Morin (2013-2017).

Plusieurs œuvres de cette dernière partie, sous un abord tout à fait paisible ou mélancolique, abritent des scènes ou détails violents qui les font basculer vers une apocalypse imminente. 1 to 87 de Fiona Tan (2014) par exemple, représente en maquette, une petite ville d'apparence paisible et bucolique que traverse un petit train électrique. 
Elle contient, quand on la regarde en détail, plusieurs scènes reflétant des maux de notre société : atteinte à l'environnement (via des forêts incendiées ou des décharges sauvages, usine polluante), misère sociale, agressions et accidents de la route. Ces scènes "destructrices" contrastent avec celles de loisirs et divertissements (cinéma en plein air, concert, camping...) qui semblent étrangères à ces catastrophes, créant une impression générale de malaise.

L'exposition se termine par la tragique scène finale du film The Truman show (1998), l'œuvre politique et déroutante de Kent Montkman Bête noire (2014) et Nostalgia de Dulce Pinzón (2011) qui représente avec humour un couple s'embrassant dans un diorama sous le regard curieux des animaux naturalisés mis à la place des visiteurs...

14 S'il est émouvant de voir ou de revoir certains dioramas historiques et en particulier les réalisations des maîtres en la matière que sont Hakeley, Hart et Ward, c'est bien le détournement des codes du diorama par les artistes contemporains qui apporte un bonus considérable à cette exposition réussie. Ils montrent à quel point ce médium muséographique a, certes, une histoire mais également un avenir, loin d'être désuet et porteur de sens. En jouant avec l'illusion de l'artificiel, il perturbe notre perception du réel. Les œuvres, souvent critiques de notre société, interrogent avec force la question du point de vue, de l'inversion des rôles et de la frontière (vitre ?) qui sépare ce qui est donné à observer et l'observateur devenu "voyeur" d'un monde mis en scène et fantasmé !

Mark Dion, Paris Streetscape, 2017

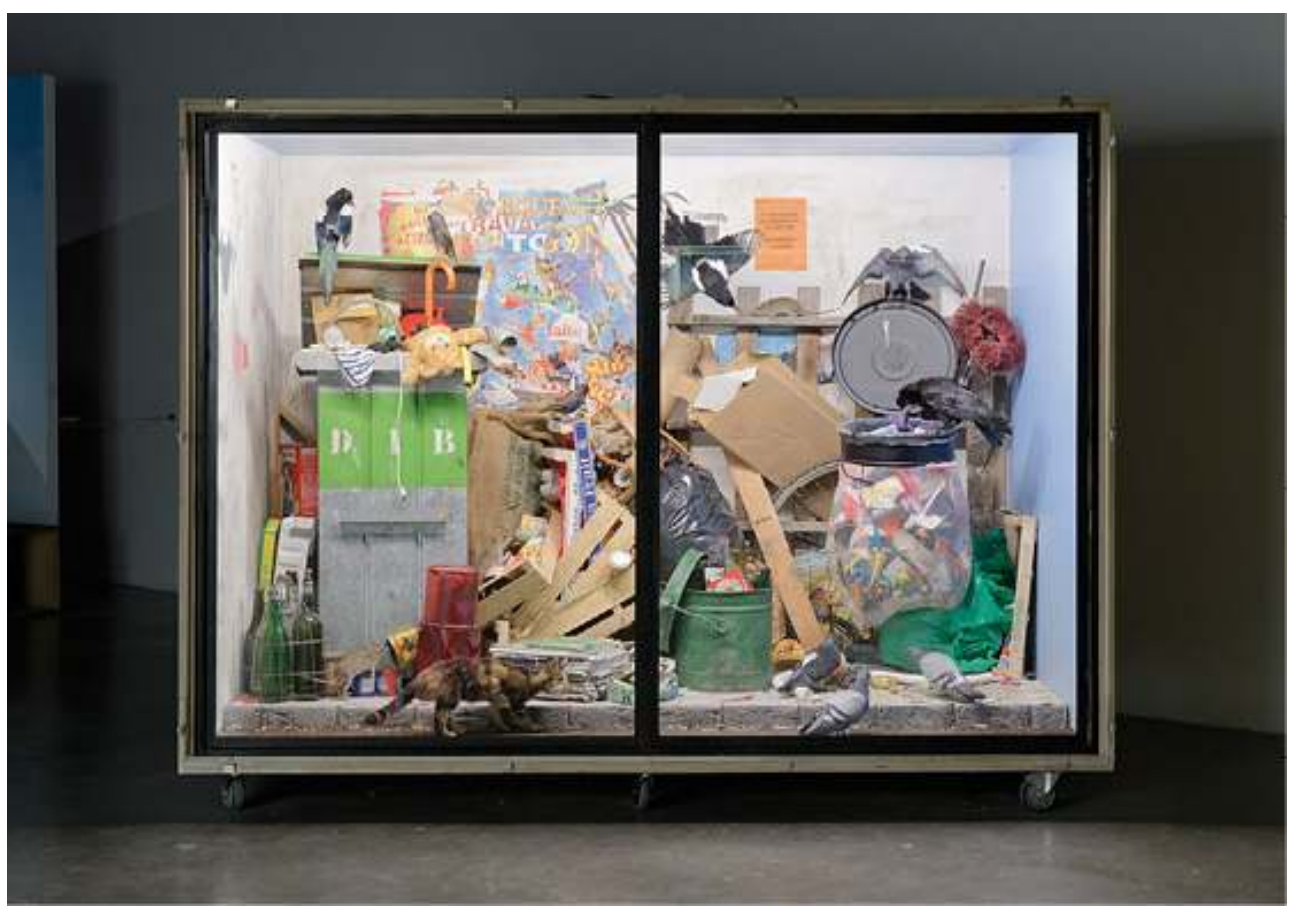

(c) Mark Dion 
Richard Barnes, Man with Buffalo, 2007

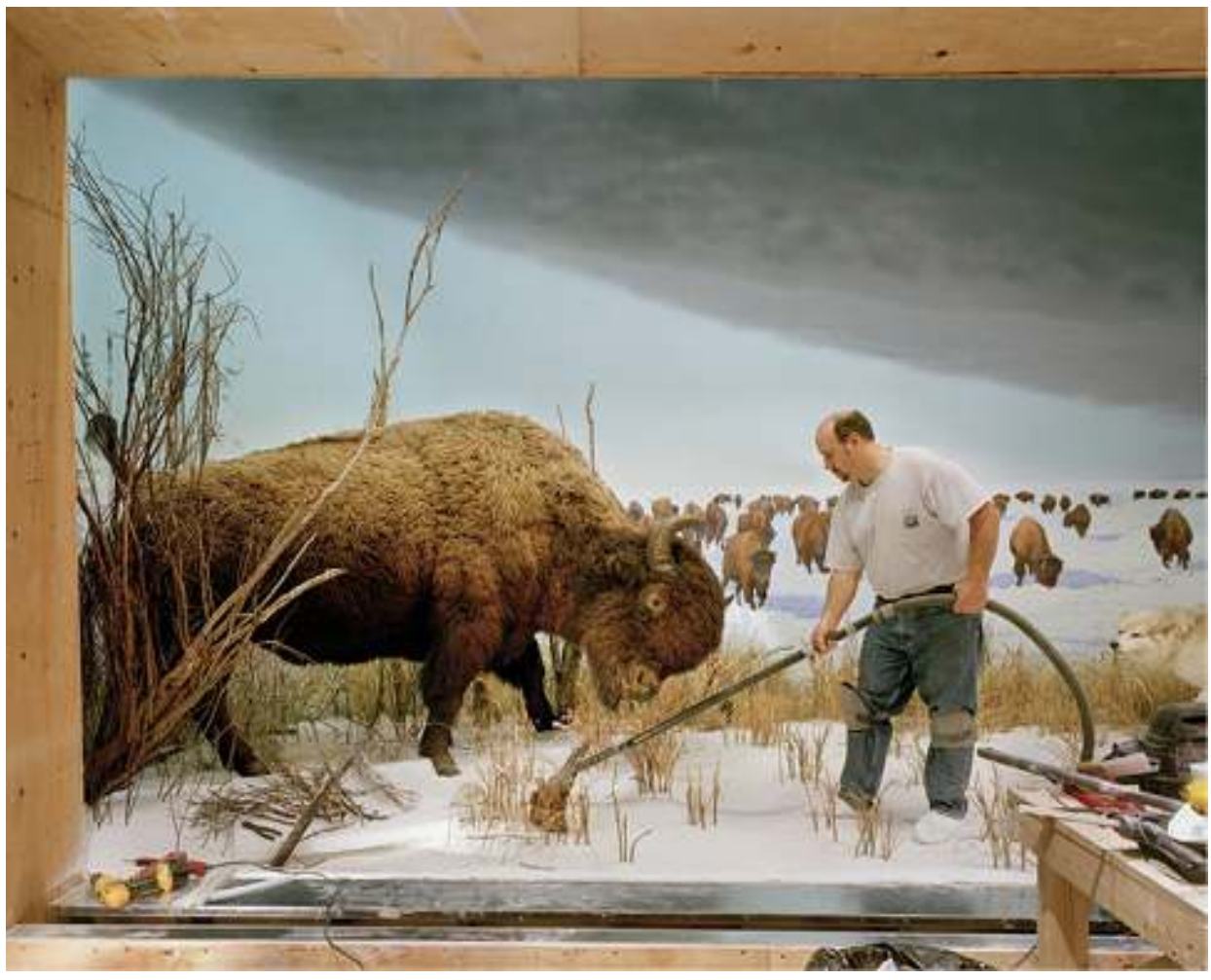

(C) Richard Barnes

Dulce Pinzon, Nostalgia, Série : "Historias del Paradiso", 2011

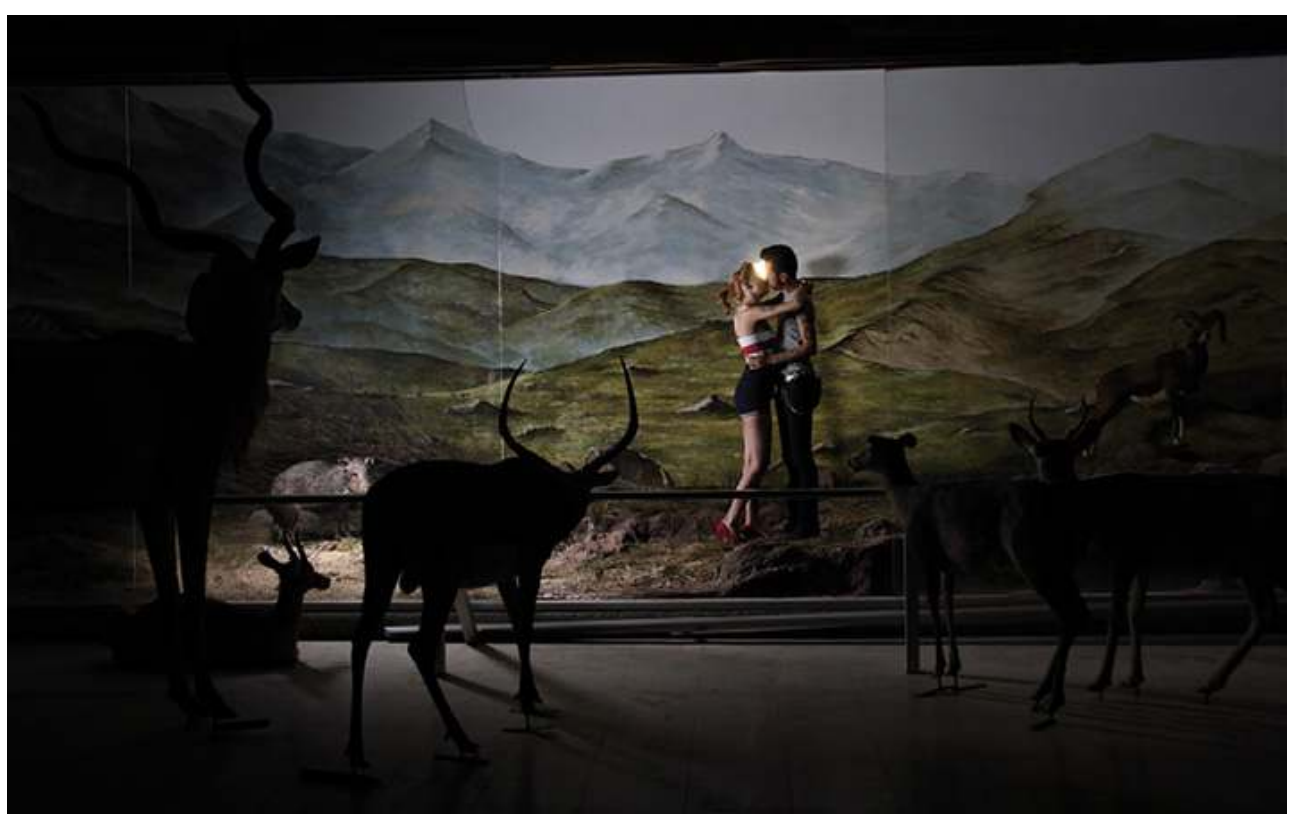

(c) Courtesy K-Echo / photos Galeria Conde (Mexico) \& H Gallery (Paris) 


\section{RÉSUMÉS}

Selon la définition communément admise le diorama est un système de présentation par mise en situation ou mise en scène d'un modèle d'exposition (un personnage historique, un animal disparu ou encore vivant à notre ère...), le faisant apparaître dans son environnement habituel. À partir de l'exposition Dioramas présentée dernièrement au Palais de Tokyo à Paris, PierreAntoine Gérard, conservateur du Muséum-Aquarium de Nancy montre la diversité des techniques et des matériaux utilisés aux différentes époques mais également la modernité de ce médium muséographique avec l'usage qu'en font aujourd'hui les artistes.

\section{INDEX}

Mots-clés : exposition, diorama, muséographie

\section{AUTEUR}

\section{PIERRE-ANTOINE GÉRARD}

conservateur du Muséum-Aquarium de Nancy 